\section{Geometric Properties of Central Catadioptric Line Images and Their Application in Calibration}

\author{
João P. Barreto and Helder Araujo
}

\begin{abstract}
In central catadioptric systems, lines in a scene are projected to conic curves in the image. This work studies the geometry of the central catadioptric projection of lines and its use in calibration. It is shown that the conic curves where the lines are mapped possess several projective invariant properties. From these properties, it follows that any central catadioptric system can be fully calibrated from an image of three or more lines. The image of the absolute conic, the relative pose between the camera and the mirror, and the shape of the reflective surface can be recovered using a geometric construction based on the conic loci where the lines are projected. This result is valid for any central catadioptric system and generalizes previous results for paracatadioptric sensors. Moreover, it is proven that systems with a hyperbolic/elliptical mirror can be calibrated from the image of two lines. If both the shape and the pose of the mirror are known, then two line images are enough to determine the image of the absolute conic encoding the camera's intrinsic parameters. The sensitivity to errors is evaluated and the approach is used to calibrate a real camera.
\end{abstract}

Index Terms-Catadioptric, omnidirectional vision, projective geometry, lines, calibration.

\section{INTRODUCTION}

APPLICATIONS that benefit from omnidirectional imagery include surveillance, 3D reconstruction, robot navigation, and visual servoing [1], [2]. The approach of combining mirrors with conventional cameras to enhance the sensor field of view is referred to as catadioptric image formation. Central catadioptric systems use specific optic-lens arrangements in order to combine two important features: a wide field of view and a single projection center. The nonlinear functions which map points in the 3D world to points in the central catadioptric image are found in [3]. This work also shows that the nonlinear mapping results in a line in the scene being projected into a conic curve. In [4], Geyer and Daniilidis propose a unifying theory for all central catadioptric systems in which conventional perspective imaging appears as a particular case. Some properties of line images are stated [4] and it is proven that a paracatadioptric sensor can be calibrated using an image of three or more lines [5].

This paper studies the geometry of line projection in central catadioptric systems. A set of projective invariant properties, most of them novel, are described and proven. These properties not only enable a deep understanding of line images, but also support the geometric constructions proposed for calibration. We show that any central catadioptric system can be fully calibrated from the image of a minimum of three lines. Moreover, if the system is hyperbolic/elliptical, then two-line projections are enough to recover the sensor parameters. The original contributions can be summarized as follows:

- A set of projective invariant properties describing the geometric relations in central catadioptric images of lines.

- A geometric construction that, given the conic loci where three or more lines are projected, is able to fully calibrate the

- The authors are with the Institute for Systems and Robotics, Department of Electrical and Computer Engineering, Polo II, University of Coimbra, 3030 Coimbra, Portugal.E-mail:\{jpbar, helder\}@isr.uc.pt.

Manuscript received 3 Feb. 2004; revised 3 Dec. 2004; accepted 16 Dec. 2004; published online 13 June 2005.

Recommended for acceptance by L. Quan.

For information on obtaining reprints of this article, please send e-mail to: tpami@computer.org, and reference IEEECS Log Number TPAMI-0065-0204. catadioptric sensor. Fully calibrate is taken to mean recovering the camera intrinsics, the relative pose between the camera and the mirror, and the type of system. The method is valid for all central catadioptric systems and generalizes previous results for paracatadioptric sensors [5].

- A method to obtain the camera intrinsics for a hyperbolic/ elliptical system using an image of a minimum of two lines in a general position. This result supports the hypothesis presented in [4] that a hyperbolic/elliptical system can be calibrated from a minimum of two lines.

Section 2 reviews the different types of central catadioptric systems and the image formation model. Section 4 describes the calibration of any central catadioptric system using three or more lines and Section 5 introduces the geometric construction to recover the camera intrinsics in a hyperbolic sensor. Our calibration method is related to the work by Liebowitz [6] for the perspective camera. Section 3 presents the projective invariant properties that give a foundation for the calibration approach. The practical application of the calibration method is discussed in Section 6. In order to show that the derived properties can be useful for reconstruction purposes, we also include an experiment where the angles between pairs of parallel lines are directly computed from catadioptric images.

\section{A Review of the Unifying Theory for Central Projection SYSTEMS}

Central catadioptric systems can be built by combining an orthographic camera with a parabolic mirror, or a conventional perspective camera with a hyperbolic, elliptical or planar mirror [7]. For the parabolic system, the distance between the camera and the mirror is not constrained. The single viewpoint constraint is satisfied whenever the camera is orthographic and the optical axis is aligned with the axis of the paraboloid. While, for the planar mirror, there is always a single effective viewpoint, in the hyperbolic and elliptical systems, the center of the perspective camera must be coincident with the outer focus of the reflective surface. In any of these three cases, the alignment between the camera and the mirror is not constrained [3]. After rotating the camera around an axis passing through its center, the catadioptric system still satisfies the single viewpoint constraint and the projection center position is kept. Finally, note that the geometry of planar catadioptric images is equivalent to conventional perspective imaging.

\subsection{The Sphere Model for Central Catadioptric Image Formation}

The mapping between points in the $3 \mathrm{D}$ world and points in the catadioptric image plane can be split into three steps, as shown in the scheme of Fig. 1 [8], [4], [3]. Consider a generic scene point $\mathbf{X}_{\mathbf{h}}$ which is projected to point $\hat{\mathbf{x}}$ in the catadioptric image plane. Each visible point can be associated with a projective ray $\mathrm{x}$ joining the point with the effective viewpoint of the system. The relation in homogeneous coordinates between $\mathbf{X}_{\mathrm{h}}$ and $\mathrm{x}$ is provided by $\mathbf{x}=\mathbf{P X}_{\mathbf{h}}$ with $\mathbf{P}=\mathbf{R}[\mathbf{I} \mid-\mathbf{C}]$. $\mathbf{P}$ is a conventional $3 \times 4$ projection matrix where $\mathbf{R}$ is the rotation matrix between world and mirror coordinate systems and $\mathbf{C}$ is the origin of the world coordinate system. Henceforth, it will be assumed, without loss of generality, that the world and sensor coordinate systems are the same and that $\mathbf{P}=[\mathbf{I} \mid \mathbf{0}]$. We think of the projective ray $\mathrm{x}$ as a point in an oriented projective plane $\mathbf{T}^{2}$. This is important when modeling panoramic vision sensors, where diametrically opposite points relative to the projection center can be imaged simultaneously. The oriented projective plane is transformed by a nonlinear function $\hbar$ (1). To each oriented point, $\mathrm{x}$ corresponds an oriented point $\overline{\mathbf{x}}$ such that $\overline{\mathbf{x}}=\hbar(\boldsymbol{x})$. The image point $\hat{\mathbf{x}}$ is obtained after a collineation $\mathbf{H}_{\mathrm{c}}\left(\hat{\mathbf{x}}=\mathbf{H}_{\mathrm{c}} \overline{\mathbf{x}}\right)$. Matrix $\mathbf{H}_{\mathrm{c}}$ is a function of the camera intrinsic parameters $\mathbf{K}_{\mathbf{c}}$, the rotation between the camera and the mirror $\mathbf{R}_{\mathbf{c}}$, and the shape of the mirror. 


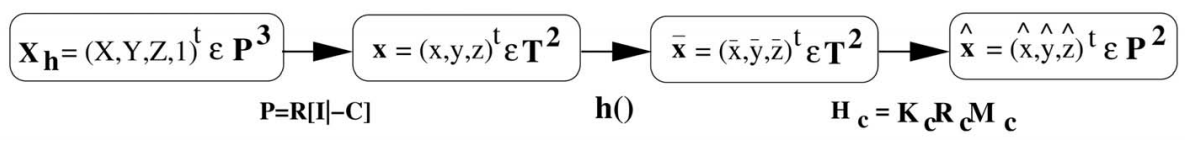

Fig. 1. Image formation in central catadioptric systems: the projection matrix $\mathbf{P}$ maps points in the world $\mathbf{X}_{\mathbf{h}}$ into points in the plane $\mathbf{x}$; function $\hbar$ denotes a nonlinear transformation between two oriented projective planes; matrix $\mathbf{H}_{c}$ represents a collineation in the plane.

TABLE 1

Parameters $\xi$ and $\psi$ ( $d$ Is the Distance between Foci and $4 p$ Is the Latus Rectum)

\begin{tabular}{|c|c|c|c|c|}
\hline & Parabolic & Hyperbolic & Elliptical & Planar \\
\hline$(\xi, \psi)$ & $(1,1+2 p)$ & $\left(\frac{d}{\sqrt{d^{2}+4 p^{2}}}, \frac{d+2 p}{\sqrt{d^{2}+4 p^{2}}}\right)$ & $\left(\frac{d}{\sqrt{d^{2}+4 p^{2}}}, \frac{d-2 p}{\sqrt{d^{2}+4 p^{2}}}\right)$ & $(0,1)$ \\
\hline
\end{tabular}

Parameters $\xi$ and $\psi$ (function $\hbar$ ) and matrix $\mathbf{M}_{\mathbf{c}}$ change according to the type of catadioptric system (Table 1).

$$
\begin{aligned}
& \hbar(\mathbf{x})=\left(x, y, z+\xi \sqrt{x^{2}+y^{2}+z^{2}}\right)^{t}, \\
& \mathbf{H}_{\mathbf{c}}=\mathbf{K}_{\mathbf{c}} \mathbf{R}_{\mathbf{c}} \underbrace{\left[\begin{array}{ccc}
\psi-\xi & 0 & 0 \\
0 & \xi-\psi & 0 \\
0 & 0 & 1
\end{array}\right]}_{\mathbf{M}_{\mathbf{c}}} .
\end{aligned}
$$

The model summarized in Fig. 1 isolates the nonlinearities of the mapping in a single function $\hbar$ which has an intuitive "concrete" interpretation. Consider the coordinate system $\Re$, with origin $\mathbf{O}$ at the effective viewpoint and an unit sphere centered in $\mathbf{O}$ (Fig. 2). Every scene point $\mathbf{X}_{\mathrm{h}}$ corresponds to an oriented ray $\mathbf{x}$ joining the $3 \mathrm{D}$ point to the effective projection center. This ray intersects the unit sphere at a single point $\mathbf{X}_{\mathrm{m}}$. Consider a point $\mathbf{O}_{\mathrm{c}}$ with coordinates $(0,0,-\xi)^{t}$ in $\Re$. For every $\mathbf{x}$, there exists a ray $\overline{\mathbf{x}}$ joining $\mathbf{O}_{\mathbf{c}}$ and point $\mathbf{X}_{\mathbf{m}}$. The nonlinear mapping $\hbar$ is isomorphic to projecting the scene in the unity sphere surface and then reprojecting the points on the sphere into a plane from a novel projection center $\mathbf{O}_{c}$ [4]. Finally, note that $\hbar$ is an injective function. One, and only one, oriented projective ray $\overline{\mathbf{x}}$ corresponds to each oriented projective ray $\mathbf{x}$. The corresponding inverse function $\hbar^{-1}$ such that $\mathbf{x}=\hbar^{-1}(\overline{\boldsymbol{x}})$ is

$$
\hbar^{-1}(\overline{\mathbf{x}})=\left[\begin{array}{c}
\frac{\bar{z} \xi+\sqrt{\bar{z}^{2}+\left(1-\xi^{2}\right)\left(\bar{x}^{2}+\bar{y}^{2}\right)}}{\bar{x}^{2}+\bar{y}^{2}+\bar{z}^{2}} \bar{x} \\
\frac{\bar{z} \xi+\sqrt{\bar{z}^{2}+\left(1-\xi^{2}\right)\left(\bar{x}^{2}+\bar{y}^{2}\right)}}{\bar{x}^{2}+\bar{y}^{2}+\bar{z}^{2}} \\
\frac{\bar{z} \xi+\sqrt{\bar{z}^{2}+\left(1-\xi^{2}\right)\left(\bar{x}^{2}+\bar{y}^{2}\right)}}{\bar{x}^{2}+\bar{y}^{2}+\bar{z}^{2}} \bar{z}-\xi
\end{array}\right]
$$

\subsection{Central Catadioptric Projection of Lines}

Consider a $3 \mathrm{D}$ line lying in a plane $\boldsymbol{\Pi}=\left(n_{x}, n_{y}, n_{z}, 0\right)^{t}$ which contains the effective viewpoint $\mathbf{O}$. From the first step of the model

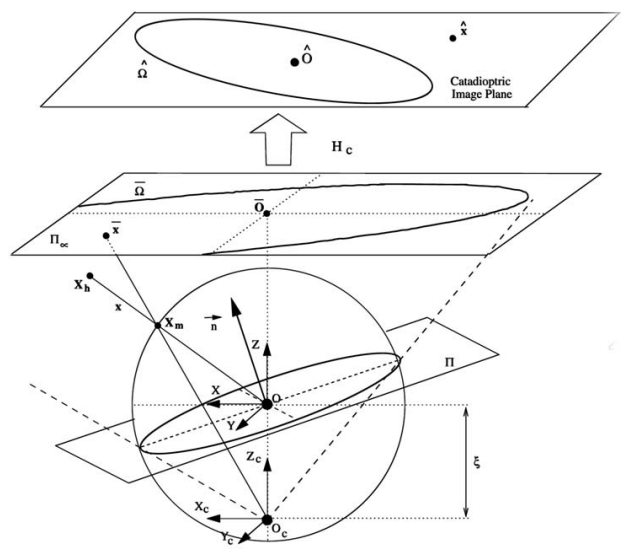

Fig. 2. The sphere model for central catadioptric image formation [4]. in Fig. 1, it follows that the 3D line projects into the 2D line $\mathbf{n}$ such that $\boldsymbol{\Pi}=\mathbf{P}^{t} \mathbf{n}$. If $\mathbf{P}=[\mathbf{I} \mid \mathbf{0}]$, then $\mathbf{n}=\left(n_{x}, n_{y}, n_{z}\right)^{t}$. Thus, the world points $\mathbf{X}_{\mathbf{h}}$, lying on the original line, are mapped into points $\mathrm{x}$ satisfying $\mathbf{n}^{\mathrm{t}} \cdot \mathbf{x}=0$. Since $\mathbf{n}^{\mathrm{t}} \cdot \mathbf{x}=0$ and $\mathbf{x}=\hbar^{-1}(\overline{\boldsymbol{x}})$, then $\boldsymbol{n}^{\boldsymbol{t}} \cdot \hbar^{-1}(\overline{\boldsymbol{x}})=0$. After some algebraic manipulation, the equality can be written in the form $\overline{\mathbf{x}}^{t} \overline{\mathbf{\Omega}} \overline{\mathbf{x}}=0$, where the conic curve $\overline{\mathbf{\Omega}}$ is shown in (4). The central catadioptric image of the line is the conic locus $\hat{\boldsymbol{\Omega}}$ obtained after the projective transformation $\mathbf{H}_{\mathbf{c}}$ (5)

$$
\begin{gathered}
\overline{\mathbf{\Omega}}=\left[\begin{array}{ccc}
n_{x}^{2}\left(1-\xi^{2}\right)-n_{z}^{2} \xi^{2} & n_{x} n_{y}\left(1-\xi^{2}\right) & n_{x} n_{z} \\
n_{x} n_{y}\left(1-\xi^{2}\right) & n_{y}^{2}\left(1-\xi^{2}\right)-n_{z}^{2} \xi^{2} & n_{y} n_{z} \\
n_{x} n_{z} & n_{y} n_{z} & n_{z}^{2}
\end{array}\right] . \\
\hat{\mathbf{\Omega}}=\mathbf{H}_{\mathbf{c}}{ }^{-t} \overline{\mathbf{\Omega}} \mathbf{H}_{\mathbf{c}}{ }^{-1} .
\end{gathered}
$$

Fig. 2 depicts the central catadioptric projection of lines using the sphere model outlined in the previous section. Plane II, containing both the line and the effective viewpoint $\mathbf{O}$, intersects the unit sphere on a great circle. The projective rays $\overline{\mathbf{x}}$, joining $\mathbf{O}_{\mathrm{c}}$ to points in the great circle, form a central cone surface. The central cone, with vertex in $\mathbf{O}_{\mathbf{c}}$, projects into the conic $\overline{\mathbf{\Omega}}$ in the canonical plane $\Pi_{\infty}$. Notice that $\overline{\mathbf{\Omega}}$ is a degenerate conic iff the imaged line is coplanar with the camera optical axis $\left(n_{z}=0\right.$ in (4)).

\section{Projective Invariant Properties of the Central Catadioptric Line Image}

Consider Fig. 3 showing the central cone formed by projective rays $\overline{\mathbf{x}}$. The cone and the canonical image plane $\Pi_{\infty}$ meet in the conic curve $\overline{\mathbf{\Omega}}$. We also know that a pencil of parallel planes intersects the plane at infinity in the same line (the horizon line), while a pencil of parallel lines in $3 \mathrm{D}$ space intersects $\Pi_{\infty}$ in a common point (the direction point). Thus, in Fig. 3, line $\overline{\boldsymbol{\pi}}$ corresponds to the horizon line of plane $\boldsymbol{\Pi}, \overline{\mathbf{D}}$ is the direction point of line $\mathbf{P}_{1} \mathbf{P}_{2}$, and $\overline{\mathbf{N}}$ is the direction orthogonal to $\Pi$. Since line $\mathbf{P}_{1} \mathbf{P}_{2}$ lies in plane $\Pi$, then $\overline{\boldsymbol{\pi}}^{t} \overline{\mathbf{D}}=0$. In addition, lines $\mathbf{P}_{1} \mathbf{P}_{2}, \mathbf{O}_{\mathrm{c}} \mathbf{O}$, projective rays $\mathbf{O}_{\mathrm{c}} \mathbf{P}_{1}, \mathbf{O}_{\mathrm{c}} \mathbf{P}_{2}$, and the normal $\tilde{\mathbf{n}}$ are coplanar and the corresponding direction points $\overline{\mathbf{D}}, \overline{\mathbf{O}}, \overline{\mathbf{P}}_{1}, \overline{\mathbf{P}}_{2}$, and $\overline{\mathbf{N}}$ are all collinear.

In general, the final catadioptric images are related to $\Pi_{\infty}$ by a projective transformation $\mathbf{H}_{\mathbf{c}}$. As shown in Fig. 3, a generic point $\overline{\mathbf{P}}$ is mapped onto $\hat{\mathbf{P}}=\mathbf{H}_{\mathbf{c}} \overline{\mathbf{P}}$, the conic curve $\overline{\mathbf{\Omega}}$ is imaged at $\hat{\mathbf{\Omega}}=\mathbf{H}_{\mathbf{c}}{ }^{-t} \overline{\boldsymbol{\Omega}} \mathbf{H}_{\mathbf{c}}{ }^{-1}$, and the line $\overline{\boldsymbol{\pi}}$ is transformed in $\hat{\boldsymbol{\pi}}=\mathbf{H}_{\mathbf{c}}{ }^{-t} \overline{\boldsymbol{\pi}}$. Collineation $\mathbf{H}_{\mathbf{c}}$ can change both the position of the line at infinity $\overline{\boldsymbol{\pi}}_{\infty}$ and the circular points $\overline{\mathbf{I}}_{\infty}, \overline{\mathbf{J}}_{\infty}$. Nevertheless, collinearity, incidence, and the cross-ratio are preserved under a projective transformation. These invariants are used to derive the projective invariant properties of a central catadioptric line image. The properties apply to any line projected onto a nondegenerate conic. In this section, we derive several properties of line images that are the basis for the calibration algorithms. The full proofs and details can be found in [8]. 


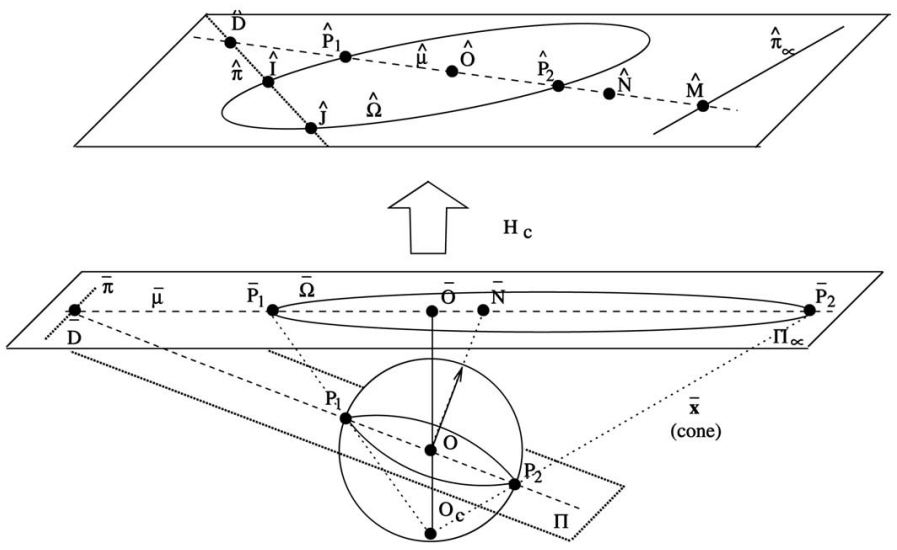

Fig. 3. Central catadioptric projection of a line lying in plane $\Pi$. The line is projected to a conic $\overline{\mathbf{\Omega}}$, which is mapped on $\hat{\mathbf{\Omega}}$ in the image.

TABLE 2

Center and Principal Axes of Conic $\bar{\Omega}$

\begin{tabular}{|ll|}
\hline Center & $\overline{\mathbf{C}}=\left(n_{x} n_{z}, n_{y} n_{z},-\left(\left(n_{x}^{2}+n_{y}^{2}\right)\left(1-\xi^{2}\right)-n_{z}^{2} \xi^{2}\right)\right)^{t}$ \\
\hline Principal Axes & $\bar{\mu}=\left(-n_{y}, n_{x}, 0\right)^{t} ; \nu=\left(\left(n_{x}^{2}+n_{y}^{2}\right)\left(1-\xi^{2}\right)-n_{z}^{2} \xi^{2}\right)\left(n_{x}, n_{y}, \frac{\left(n_{x}^{2}+n_{y}^{2}\right) n_{z}}{\left(n_{x}^{2}+n_{y}^{2}\right)\left(1-\xi^{2}\right)-n_{z}^{2} \xi^{2}}\right)^{t}$ \\
\hline Major and Minor Axes & $\frac{\sqrt{n_{x}^{2}+n_{y}^{2}+n_{z}^{2}}}{\left.n_{x}^{2}+n_{y}^{2}\right)\left(1-\xi^{2}\right)-n_{z}^{2} \xi^{2} \mid} ; \frac{n_{z}^{2} \sqrt{n_{x}^{2}+n_{y}^{2}+n_{z}^{2}} \xi^{2}}{\left.\left(n_{x}^{2}+n_{y}^{2}\right)\left(1-\xi^{2}\right)-n_{z}^{2} \xi^{2}\right)^{2}}$ \\
\hline
\end{tabular}

\subsection{Catadioptric Projection of a Single Line}

As shown in Fig. 3, plane $\boldsymbol{\Pi}=\left(n_{x}, n_{y}, n_{z}, 0\right)^{t}$ intersects the sphere in a great circle which is projected on conic $\overline{\mathbf{\Omega}}$. The point conic $\overline{\mathbf{\Omega}}$ is transformed in $\hat{\mathbf{\Omega}}=\mathbf{H}_{\mathrm{c}}{ }^{-t} \overline{\mathbf{\Omega}} \mathbf{H}_{\mathbf{c}}{ }^{-1}$, which is the central catadioptric image of any line lying in $\boldsymbol{\Pi}$. The principal point $\overline{\mathbf{O}}=(0,0,1)^{t}$ and the normal direction $\overline{\mathbf{N}}=\left(n_{x}, n_{y}, n_{z}\right)^{t}$ are mapped in points $\hat{\mathbf{O}}$ and $\hat{\mathbf{N}}$ by collineation $\mathbf{H}_{\mathrm{c}}$. In a similar way, the horizon line $\overline{\boldsymbol{\pi}}$ is mapped on $\hat{\boldsymbol{\pi}}$ and the image of the absolute conic $\overline{\boldsymbol{\Omega}}_{\infty}$ (not depicted) is $\hat{\mathbf{\Omega}}_{\infty}=\mathbf{H}_{\mathbf{c}}{ }^{-t} \mathbf{H}_{\mathbf{c}}{ }^{-1}$.

Proposition 1. If the point conic $\hat{\mathbf{\Omega}}$ is a line image, then the polar of the image center $\hat{\mathbf{O}}$ with respect to $\hat{\mathbf{\Omega}}$ is the horizon line $\hat{\boldsymbol{\pi}}$ of the plane $\boldsymbol{\Pi}$ containing the original line $(\hat{\boldsymbol{\pi}}=\hat{\mathbf{\Omega}}$. $\hat{\mathbf{O}})$.

Proof. Plane $I$ intersects the unit sphere in a great circle. Assume a line with direction $\overline{\mathbf{D}}$ passing through $\mathbf{O}$ and lying in $\Pi$. This line intersects the great circle at two antipodal points $\mathbf{P}_{1}$ and $\mathbf{P}_{2}$ which are equidistant to $\mathbf{O}$. Since $\overline{\boldsymbol{\pi}}$ is the intersection of $\boldsymbol{\Pi}$ with the plane at infinity, then $\mathbf{O}$ and $\overline{\boldsymbol{\pi}}$ are pole/polar with respect to the great circle. This relationship is preserved by the projection onto $\Pi_{\infty}$ and by the projective transformation $\mathbf{H}_{\mathbf{c}}$. $\square$

Proposition 2. If $\hat{\boldsymbol{\Omega}}$ is the catadioptric image of a line, then the polar line $\hat{\boldsymbol{\pi}}$ of the image center $\hat{\boldsymbol{O}}(\hat{\boldsymbol{\pi}}=\hat{\mathbf{\Omega}}$. $\hat{\mathbf{O}})$ intersects the conic locus $\hat{\mathbf{\Omega}}$ in two points $\hat{\mathbf{I}}$ and $\hat{\mathbf{J}}$ which lie on the image of the absolute conic $\hat{\mathbf{\Omega}}_{\infty}$.

Proof. The great circle on the sphere intersects the horizon line $\overline{\boldsymbol{\pi}}$ in a pair of circular points $\overline{\mathbf{I}}, \overline{\mathbf{J}}$, which lie on the absolute conic $\overline{\mathbf{\Omega}}_{\infty}$. Since plane $\boldsymbol{\Pi}$ cuts the central cone with vertex in $\mathbf{O}_{\mathbf{c}}$ in a circular section, then $\overline{\mathbf{\Omega}}$ and $\overline{\mathbf{\Omega}}_{\infty}$ intersect at points $\overline{\mathbf{I}}, \overline{\mathbf{J}}$. The established relations hold in the catadioptric image plane after the projective transformation $\mathbf{H}_{\mathbf{c}}$.

Table 2 provides the equations of the center $\overline{\mathbf{C}}$ and the principal axes $\overline{\boldsymbol{\mu}}, \overline{\boldsymbol{\nu}}$ of the conic curve $\overline{\boldsymbol{\Omega}}$ lying in plane $\boldsymbol{\Pi}_{\infty}$. The computation is straightforward, bearing in mind that the line at infinity $\overline{\boldsymbol{\pi}}_{\infty}$ and the circular points $\overline{\mathbf{I}}_{\infty}, \overline{\mathbf{J}}_{\infty}$ are in the canonical position. As stated, $\boldsymbol{\Pi}_{\infty}$ and the final image are related by a collineation $\mathbf{H}_{\mathbf{c}}$. Points $\hat{\mathbf{I}}_{\infty}, \hat{\mathbf{J}}_{\infty}$ are the circular points in the catadioptric image plane, iff $\mathbf{H}_{\mathbf{c}}$ is a similarity transformation. If this is not the case, then the transformed lines $\hat{\boldsymbol{\mu}}, \hat{\boldsymbol{\nu}}$ are no longer the principal axes of $\hat{\boldsymbol{\Omega}}$. Similarly, point $\hat{\mathbf{C}}$ is the center of conic $\hat{\boldsymbol{\Omega}}$ iff $\mathbf{H}_{\mathrm{c}}$ is an affine transformation such that $\hat{\boldsymbol{\pi}}_{\infty}=\mathbf{H}_{\mathbf{c}}{ }^{-t} \overline{\boldsymbol{\pi}}_{\infty}$ is still the line at infinity. The following proposition makes it possible to determine the position of line $\hat{\boldsymbol{\mu}}$ for an arbitrary collineation $\mathbf{H}_{\mathbf{c}}$ when points $\hat{\mathbf{O}}$ and $\hat{\mathbf{N}}$ are known.

Proposition 3. The image points $\hat{\mathbf{O}}$ and $\hat{\mathbf{N}}$ define line $\hat{\boldsymbol{\mu}}$, which is the locus where the major axis $\overline{\boldsymbol{\mu}}$ of the conic curve $\overline{\boldsymbol{\Omega}}$ is mapped by collineation $\mathbf{H}_{\mathbf{c}}$

Proof. Both the principal point $\overline{\mathbf{O}}=(0,0,1)^{t}$ and the normal direction $\overline{\mathbf{N}}=\left(n_{x}, n_{y}, n_{z}\right)^{t}$ lie on the major axis $\overline{\boldsymbol{\mu}}=\left(-n_{y}, n_{x}, 0\right)^{t}$ (see Table 2). The scheme given in Fig. 3 shows that $\overline{\boldsymbol{\mu}}$ is the intersection between the reference plane $\boldsymbol{\Pi}_{\infty}$ and the plane containing both the normal direction $\tilde{\mathbf{n}}$ and the $\mathrm{Z}$ axis. Since collineation $\mathbf{H}_{c}$ preserves incidence and collinearity, then points $\hat{\mathbf{O}}$ and $\hat{\mathbf{N}}$ must lie on the locus $\hat{\boldsymbol{\mu}}$, where $\overline{\boldsymbol{\mu}}$ is mapped. However, it should be remembered that line $\hat{\boldsymbol{\mu}}$ is no longer the major axis of the catadioptric line image $\hat{\mathbf{\Omega}}$.

Corollary $\mathbf{1}$. The pole of $\hat{\boldsymbol{\mu}}$ with respect to the point conic $\hat{\mathbf{\Omega}}$ lies on line $\hat{\boldsymbol{\pi}}_{\infty}$, where the line at infinity $\overline{\boldsymbol{\pi}}_{\infty}$ is mapped.

Consider, on the reference plane $\boldsymbol{\Pi}_{\infty}$, the principal point $\overline{\mathbf{O}}=(0,0,1)^{t}$, the normal direction $\overline{\mathbf{N}}=\left(n_{x}, n_{y}, n_{z}\right)^{t}$, and the conic center $\overline{\mathbf{C}}$ (Table 2). The horizon line $\overline{\boldsymbol{\pi}}=\overline{\mathbf{\Omega}} \overline{\mathbf{O}}$ (Proposition 1) intersects the major axis $\overline{\boldsymbol{\mu}}$ (Table 2) at point $\overline{\mathbf{D}}$. It can be shown by direct calculation that the crossratio $\{\overline{\mathbf{O}}, \overline{\mathbf{D}} ; \overline{\mathbf{N}}, \overline{\mathbf{C}}\}$ is always equal to the square of the parameter $\xi$ (Table 1). Since the cross-ratio is a projective invariant, it follows that:

Proposition 4 . The cross ratio between points $\hat{\boldsymbol{O}}, \hat{\mathbf{N}}, \hat{\mathbf{D}}$, and $\hat{\mathbf{C}}$, lying on $\hat{\boldsymbol{\mu}}$, depends only on the shape of the reflective surface used in the central catadioptric system. In particular, $\{\hat{\mathbf{O}}, \hat{\mathbf{D}} ; \hat{\mathbf{N}}, \hat{\mathbf{C}}\}=\xi^{2}$.

The conic center $\overline{\mathbf{C}}$ is, by definition, the pole of the line at infinity $\overline{\boldsymbol{\pi}}_{\boldsymbol{\infty}}=(0,0,1)^{t}$ with respect to $\overline{\boldsymbol{\Omega}}$. Thus, if both $\hat{\boldsymbol{\Omega}}$ and $\hat{\boldsymbol{\pi}}_{\boldsymbol{\infty}}$ are known, then the image point where $\overline{\mathbf{C}}$ is mapped is $\hat{\mathbf{C}}=\hat{\mathbf{\Omega}}^{*} \cdot \hat{\boldsymbol{\pi}}_{\infty}$ with $\hat{\mathbf{\Omega}}^{*}$ standing for the dual conic envelope.

The cross-ratio relation of (6) is used in Section 5 and it can be proven in the same way as Proposition 4 . Both $\hat{\mathbf{M}}$ and $\hat{\boldsymbol{N}}^{*}$ lie on 

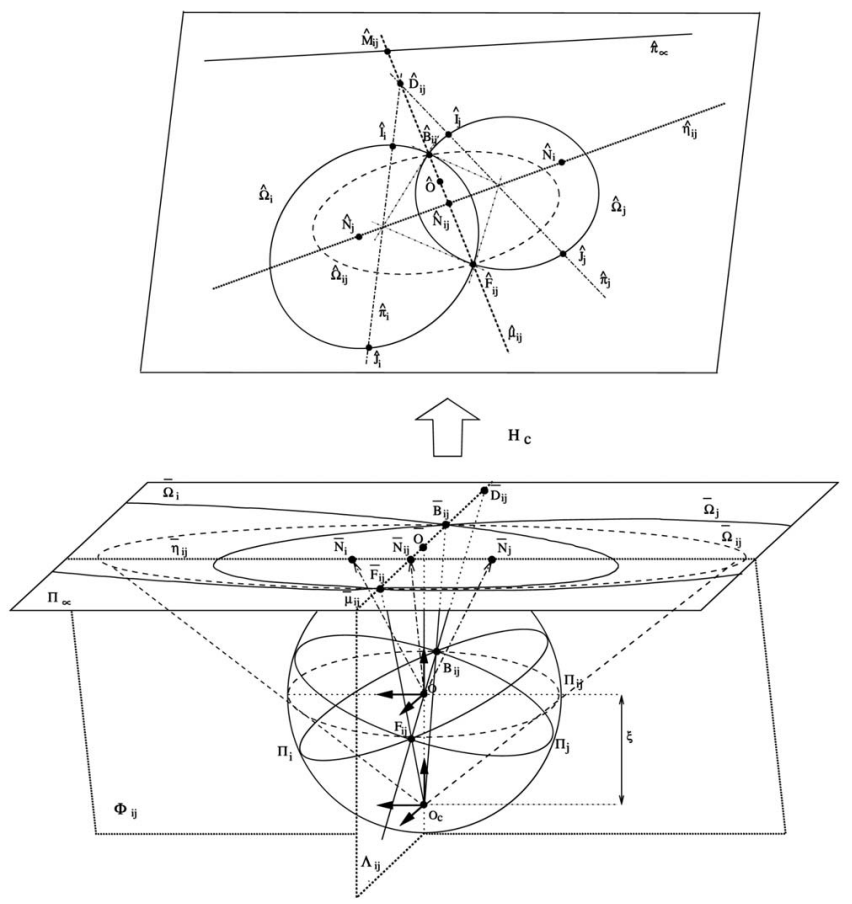

Fig. 4. The central catadioptric image of a pair of lines. Projection of points from the unit sphere to the plane at infinity and the projective transformation $\mathbf{H}_{\mathbf{c}}$ from $\Pi_{\infty}$ into the catadioptric image plane.

line $\hat{\boldsymbol{\mu}}$. While $\hat{\mathbf{M}}$ is the point where lines $\hat{\boldsymbol{\mu}}$ and $\overline{\boldsymbol{\pi}}_{\infty}$ meet, point $\hat{\boldsymbol{N}}^{*}$ is the intersection of $\hat{\boldsymbol{\mu}}$ with the polar line of $\hat{\mathbf{N}}$ (Fig. 3).

$$
\{\hat{\boldsymbol{C}}, \hat{\boldsymbol{N}} ; \hat{\boldsymbol{M}}, \hat{\boldsymbol{O}}\}=\xi^{2}-\frac{2 \xi^{2}\left\{\hat{\boldsymbol{N}}^{*}, \hat{\boldsymbol{N}} ; \hat{\boldsymbol{M}}, \hat{\boldsymbol{C}}\right\}}{\left(1-\xi^{2}\right)\left(1+\sqrt{1+\frac{4 \xi^{2}\left\{\hat{\boldsymbol{N}}^{*}, \hat{\boldsymbol{N}} ; \hat{\boldsymbol{M}}, \hat{\boldsymbol{C}}\right\}}{\left(1-\xi^{2}\right)^{2}}}\right)} .
$$

\subsection{Parabolic Projection of a Single Line}

Proposition 5 is only valid for catadioptric systems combining a parabolic mirror with an orthographic camera. In this case, parameter $\xi$ is unitary (Table 1) and the projection from the unitary sphere onto the reference plane $\boldsymbol{\Pi}_{\infty}$ is a stereographic projection [4]. The result of Proposition 5 is used in Section 4 to explain why the paracatadioptric sensor can not be calibrated using the image of two lines. The interested reader can find the proof of the statement in [8].

Proposition 5. In a central paracatadioptric image, point $\hat{\mathbf{N}}$ and line $\hat{\boldsymbol{\pi}}_{\infty}$ are pole/polar with respect to the corresponding line image $\hat{\mathbf{\Omega}}$.

\subsection{Catadioptric Projection of a Pair of Lines}

Fig. 4 shows the central catadioptric projection of a pair of lines. Two lines, lying in planes $\boldsymbol{\Pi}_{i}$ and $\boldsymbol{\Pi}_{j}$, are imaged in conics $\hat{\boldsymbol{\Omega}}_{\boldsymbol{i}}$ and $\hat{\boldsymbol{\Omega}}_{\boldsymbol{j}}$. Both $\Pi_{i}$ and $\Pi_{j}$ pass through the effective viewpoint $\mathbf{O}$, and cut the unit sphere in two great circles with a common pair of antipodal points $\mathbf{F}_{\mathrm{ij}}, \mathbf{B}_{\mathrm{ij}}$. The circles define two central cones with vertices in $\mathbf{O}_{\mathbf{c}}$, which intersect the reference plane $\boldsymbol{\Pi}_{\infty}$ in $\overline{\boldsymbol{\Omega}}_{\boldsymbol{i}}$ and $\overline{\boldsymbol{\Omega}}_{\boldsymbol{j}}$. These conic curves meet in a pair of real points $\overline{\mathbf{F}}_{\mathrm{ij}}, \overline{\mathbf{B}}_{\mathrm{ij}}$, which are the projections of the antipodal points. The line passing through these points is $\overline{\boldsymbol{\mu}}_{i j}=\overline{\mathbf{F}}_{\mathbf{i j}} \wedge \overline{\mathbf{B}}_{\mathbf{i j}}$. The antipodal points define a 3D line $\mathbf{F}_{\mathbf{i j}} \mathbf{B}_{\mathbf{i j}}$ with direction $\overline{\mathbf{D}}_{\mathrm{ij}}$. Assume $\boldsymbol{\Phi}_{i j}$ as the plane orthogonal to direction $\overline{\mathbf{D}}_{\mathrm{ij}}$ and passing through the origin $\mathbf{O}$. Since $\overline{\mathbf{D}}_{\mathrm{ij}}$ is a common direction of $\Pi_{i}$ and $\Pi_{j}$, then the normal vectors $\tilde{\mathbf{n}}_{\mathbf{i}}$ and $\overrightarrow{\mathbf{n}}_{\mathbf{j}}$ must lie in plane $\boldsymbol{\Phi}_{i j}$. It follows that line $\overline{\boldsymbol{\eta}}_{\boldsymbol{i} \boldsymbol{j}}$, which is the intersection of $\boldsymbol{\Phi}_{\boldsymbol{i} \boldsymbol{j}}$ and $\boldsymbol{\Pi}_{\infty}$, passes through the direction points $\overline{\mathbf{N}}_{\mathbf{i}}$ and $\overline{\mathbf{N}}_{\mathbf{j}}$. Curves $\overline{\boldsymbol{\Omega}}_{\boldsymbol{i}}$ and $\overline{\boldsymbol{\Omega}}_{\boldsymbol{j}}$ are mapped by collineation $\mathbf{H}_{\mathrm{c}}$ onto conics $\hat{\boldsymbol{\Omega}}_{\boldsymbol{i}}$ and $\hat{\boldsymbol{\Omega}}_{j}$. Likewise, points $\overline{\mathbf{F}}_{\mathrm{ij}}, \overline{\mathbf{B}}_{\mathrm{ij}}, \overline{\mathbf{D}}_{\mathrm{ij}}, \overline{\mathbf{N}}_{\mathrm{i}}, \overline{\mathbf{N}}_{\mathrm{j}}$ are transformed to points $\hat{\mathbf{F}}_{\mathrm{ij}}, \hat{\mathbf{B}}_{\mathrm{ij}}, \hat{\mathbf{D}}_{\mathrm{ij}}, \hat{\mathbf{N}}_{\mathrm{i}}$,
$\hat{\mathbf{N}}_{\mathbf{j}}$, and lines $\overline{\boldsymbol{\mu}}_{i j}$ and $\overline{\boldsymbol{\eta}}_{\boldsymbol{i j}}$ are mapped to $\hat{\boldsymbol{\mu}}_{\boldsymbol{i} \boldsymbol{j}}$ and $\hat{\boldsymbol{\eta}}_{\boldsymbol{i} \boldsymbol{j}}$. Proposition 6, stated in [4], relates the intersection points of two catadioptric line projections with the image center.

Proposition 6. If $\hat{\boldsymbol{F}}_{\boldsymbol{i j}}$ and $\hat{\mathbf{B}}_{\mathrm{ij}}$ are the intersection points of two catadioptric line images $\hat{\mathbf{\Omega}}_{\boldsymbol{i}}$ and $\hat{\mathbf{\Omega}}_{\boldsymbol{j}}$, then the image center $\hat{\mathbf{O}}$ is always collinear with $\hat{\mathbf{F}}_{\mathrm{ij}}$ and $\hat{\mathbf{B}}_{\mathrm{ij}}$.

As shown in Fig. 4 , the line $\mathbf{F}_{\mathrm{ij}} \mathbf{B}_{\mathbf{i j}}$ is common to planes $\boldsymbol{\Pi}_{\boldsymbol{i}}, \boldsymbol{\Pi}_{\mathbf{j}}$, and $\boldsymbol{\Lambda}_{\boldsymbol{i} j}$ and the vanishing lines $\overline{\boldsymbol{\pi}}_{\boldsymbol{i}}, \overline{\boldsymbol{\pi}}_{\boldsymbol{j}}$, and $\overline{\boldsymbol{\mu}}_{\boldsymbol{i}}$ must intersect in the direction point $\overline{\mathbf{D}}_{\mathrm{ij}}$. The projective transformation $\mathbf{H}_{\mathrm{c}}$ preserves incidence, which means that lines $\hat{\boldsymbol{\pi}}_{\boldsymbol{i}}, \hat{\boldsymbol{\pi}}_{\boldsymbol{j}}$ and $\hat{\boldsymbol{\mu}}_{\boldsymbol{i}}$ intersect in a single point $\hat{\mathbf{D}}_{\mathrm{ij}}$. The proof of Corollary 2 is based on this property (for details, see [8]). If the pair of imaged lines is parallel, then the common direction $\overline{\mathbf{D}}_{\mathrm{ij}}$ of the corresponding planes $\boldsymbol{\Pi}_{\boldsymbol{i}}$ and $\boldsymbol{\Pi}_{j}$ is the direction of the parallel lines. This result is useful for reconstruction tasks such as the one described in [1]. It implicitly states that it is possible to directly recover the orientation of any world plane from the calibrated image of two sets of parallel lines lying in it.

Corollary 2. Consider two parallel lines projected in the catadioptric image plane on conics $\hat{\mathbf{\Omega}}_{\boldsymbol{i}}$ and $\hat{\mathbf{\Omega}}_{\boldsymbol{j}}$. If the polar lines of $\hat{\mathbf{O}}$ are, respectively, $\hat{\boldsymbol{\pi}}_{\boldsymbol{i}}$ and $\hat{\boldsymbol{\pi}}_{\boldsymbol{j}}$, then the direction of the parallel lines is $\overline{\mathbf{D}}_{\mathrm{ij}}=\mathbf{H}_{\mathrm{c}}{ }^{-1}\left(\hat{\boldsymbol{\pi}}_{\boldsymbol{i}} \wedge \hat{\boldsymbol{\pi}}_{\boldsymbol{j}}\right)$.

The pair of conics $\hat{\boldsymbol{\Omega}}_{\boldsymbol{i}}$ and $\hat{\boldsymbol{\Omega}}_{\boldsymbol{j}}$ are the images of two lines lying, respectively, in planes $\Pi_{i}$ and $\boldsymbol{\Pi}_{\boldsymbol{j}}$. Any pair of catadioptric line images has a line $\hat{\boldsymbol{\mu}}_{\boldsymbol{i} j}$ and $\hat{\boldsymbol{\eta}}_{\boldsymbol{i} j}$ associated with it (Fig. 4). Assume the pencil of planes $\boldsymbol{\Pi}$ defined by the line passing through the antipodal points $\mathbf{F}_{\mathrm{ij}}$ and $\mathbf{B}_{\mathrm{ij}}$. Any line lying in one of these planes is projected onto a conic curve $\hat{\mathbf{\Omega}}$ passing through $\hat{\mathbf{F}}_{\mathrm{ij}}$ and $\hat{\mathbf{B}}_{\mathrm{ij}}$. Moreover, since the vectors normal to the pencil of planes are contained in $\boldsymbol{\Phi}_{i j}$, then the corresponding direction points $\overline{\mathbf{N}}$ are mapped on line $\hat{\boldsymbol{\eta}}_{i j}$. Proposition 7 states an important property verified by any $\hat{\boldsymbol{\Omega}}$ belonging to the family of catadioptric line images defined by the intersection points of $\hat{\mathbf{\Omega}}_{\boldsymbol{i}}$ and $\hat{\mathbf{\Omega}}_{\boldsymbol{j}}$.

Proposition 7. If conic $\hat{\mathbf{\Omega}}$ is a line image passing through points $\hat{\mathbf{F}}_{\mathbf{i j}}$ and $\hat{\mathbf{B}}_{\mathrm{ij}}$, then the pole of $\hat{\boldsymbol{\mu}}_{\boldsymbol{i j}}$ with respect to $\hat{\mathbf{\Omega}}$ lies on $\hat{\boldsymbol{\eta}}_{\boldsymbol{i j}}$.

Proof. Point $\hat{\mathbf{Y}}$ is the pole, with respect to $\hat{\mathbf{\Omega}}$, of line $\hat{\boldsymbol{\mu}}_{i j}$ passing through $\hat{\mathbf{F}}_{\mathrm{ij}}, \hat{\mathbf{B}}_{\mathrm{ij}}\left(\hat{\mathbf{Y}}=\hat{\mathbf{\Omega}}^{*} \cdot \hat{\boldsymbol{\mu}}_{i j}\right)$. In order to prove the statement, we must show that $\hat{\boldsymbol{\eta}}_{\boldsymbol{i j}}$ contains $\hat{\mathbf{Y}}$. The polar line of the image center $\hat{\mathbf{O}}$ with respect to $\hat{\boldsymbol{\Omega}}$ is $\hat{\boldsymbol{\pi}}$. Since $\hat{\mathbf{O}}$ lies on $\hat{\boldsymbol{\mu}}_{\boldsymbol{i j}}$ (Proposition 6), then point $\hat{\mathbf{Y}}$ must lie on $\hat{\boldsymbol{\pi}}$ [9]. Line $\hat{\boldsymbol{\pi}}$ intersects $\hat{\boldsymbol{\mu}}_{i j}$ at point $\hat{\mathbf{D}}_{\mathrm{ij}}$, and the conic $\hat{\boldsymbol{\Omega}}$ at points $\hat{\mathbf{I}}$ and $\hat{\mathbf{J}}$. Since $\hat{\mathbf{Y}}$ and $\hat{\boldsymbol{\mu}}_{i j}$ are pole/polar, then the pairs of points $\{\hat{\mathbf{I}}, \hat{\mathbf{J}}\}$ and $\left\{\hat{\mathbf{D}}_{\mathrm{ij}}, \hat{\mathbf{Y}}\right\}$ are harmonic conjugates [9]. From Proposition 1, it follows that $\hat{\boldsymbol{\pi}}$ is the locus of points where the horizon line of plane $\Pi$ is mapped. Plane $\Pi$, containing the imaged line, belongs to the pencil defined by $\Pi_{i}$ and $\Pi_{j}$ (Fig. 4). The common direction $\overline{\mathbf{D}}_{\mathrm{ij}}$ is orthogonal to plane $\boldsymbol{\Phi}_{i j}$, whose vanishing line is $\overline{\boldsymbol{\eta}}_{\boldsymbol{i} \boldsymbol{j}}$. Thus, if the absolute conic is mapped on $\hat{\boldsymbol{\Omega}}_{\infty}$ by $\mathbf{H}_{\mathrm{c}}$, then $\hat{\mathbf{D}}_{\mathrm{ij}}$ and $\hat{\boldsymbol{\eta}}_{\boldsymbol{i} j}$ are pole/polar with respect to $\hat{\boldsymbol{\Omega}}_{\infty}$. Line $\hat{\boldsymbol{\pi}}$ intersects conics $\hat{\mathbf{\Omega}}_{\infty}$ and $\hat{\mathbf{\Omega}}$ at the same pair of points $\hat{\mathbf{I}}, \hat{\mathbf{J}}$ (Proposition 2). Assume that lines $\hat{\boldsymbol{\pi}}$ and $\hat{\boldsymbol{\eta}}_{\boldsymbol{i}}$ intersect at point $\hat{\mathbf{K}}$. Since $\hat{\mathbf{D}}_{\mathrm{ij}}$ and $\hat{\eta}_{\mathrm{ij}}$ are pole/polar with respect to $\hat{\mathbf{\Omega}}_{\infty}$, then the pairs of points $\{\hat{\mathbf{I}}, \hat{\mathbf{J}}\}$ and $\left\{\hat{\mathbf{D}}_{\mathrm{ij}}, \hat{\mathbf{K}}\right\}$ are also harmonic conjugates. Therefore, points $\hat{\mathbf{Y}}$ and $\hat{\mathbf{K}}$ must be the coincident.

\section{Calibration of Central Catadioptric System USING THE IMAGE OF $K$ LINES}

The geometric construction summarized in Table 3 calibrates any central catadioptric system from the image of $K \geq 3$ lines. The lines must project onto nondegenerate conics that do not intersect in the same pair of points. The method is illustrated in Fig. 5 for the minimum number of $K=3$ lines. Consider conics $\hat{\mathbf{\Omega}}_{\mathbf{1}}, \hat{\mathbf{\Omega}}_{\mathbf{2}}$, and $\hat{\mathbf{\Omega}}_{\mathbf{3}}$ corresponding to the catadioptric projection of three lines. As shown in Fig. 5a, each pair of conics $\hat{\mathbf{\Omega}}_{\boldsymbol{i}}, \hat{\mathbf{\Omega}}_{\boldsymbol{j}}$ intersects in two real points which define a line $\hat{\boldsymbol{\mu}}_{i j}(i j=12,13,23)$. According to Proposition 6, lines $\hat{\boldsymbol{\mu}}_{\mathbf{1 2}}, \hat{\boldsymbol{\mu}}_{\mathbf{1 3}}$, and $\hat{\boldsymbol{\mu}}_{\mathbf{1 2}}$ must intersect at the image center $\hat{\mathbf{O}}$. The polar line $\hat{\boldsymbol{\pi}}_{\boldsymbol{i}}=\hat{\mathbf{\Omega}}_{\boldsymbol{i}} \hat{\mathbf{O}}$ intersects the conic curve at 
TABLE 3

Calibrating a Central Catadioptric System Using $K$ Lines $(K \geq 3)$

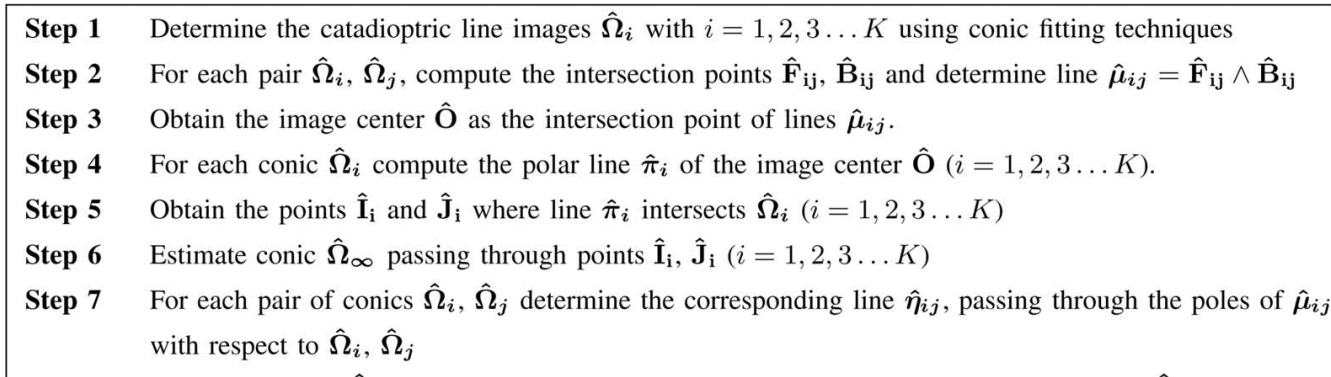

Step 8 Each line image $\hat{\Omega}_{i}$ has a pencil of lines $\hat{\eta}_{i j}$ associated with it. Determine the point $\hat{\mathrm{N}}_{\mathrm{i}}$ where those lines intersect $(i=1,2,3 \ldots K)$

Step 9 Determine the line $\hat{\boldsymbol{\mu}}_{i}=\hat{\mathbf{N}}_{\mathbf{i}} \wedge \hat{\mathbf{O}}$ associated with the catadioptric line image $\hat{\boldsymbol{\Omega}}_{i}(i=1,2,3 \ldots K)$

Step 10 Estimate the locus of the line at infinity $\hat{\pi}_{\infty}$ knowing that it goes through the poles of $\hat{\mu}_{i}$ with respect to conic $\hat{\Omega}_{i}(i=1,2,3 \ldots K)$

Step 11 For each conic $\hat{\Omega}_{i}$ determine the intersection point $\hat{\mathbf{D}}_{\mathbf{i}}=\hat{\mu}_{\mathbf{i}} \wedge \hat{\pi}_{i}$ and the pole $\hat{\mathbf{C}}_{\mathbf{i}}=\hat{\mathbf{\Omega}}_{i}^{*} \cdot \hat{\pi}_{\infty}$ which must lie on line $\hat{\boldsymbol{\mu}}_{i}(i=1,2,3 \ldots K)$

Step 12 The mirror parameter $\xi$ is provided by $\sqrt{\left\{\hat{\mathbf{O}}, \hat{\mathbf{D}}_{i} ; \hat{\mathbf{N}}_{\mathbf{i}}, \hat{\mathbf{C}}_{\mathbf{i}}\right\}}(i=1,2,3 \ldots K)$

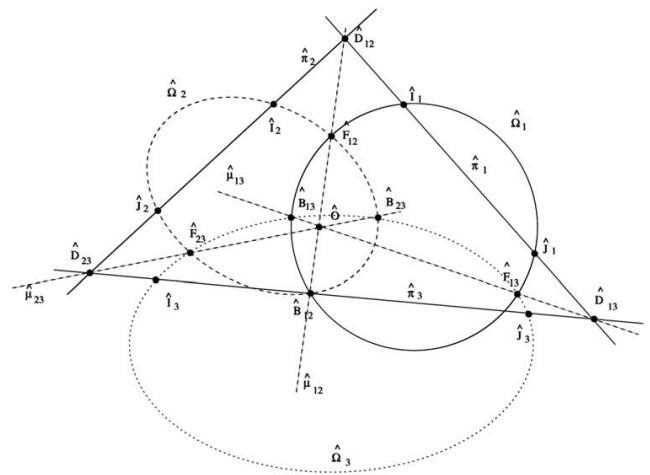

(a)

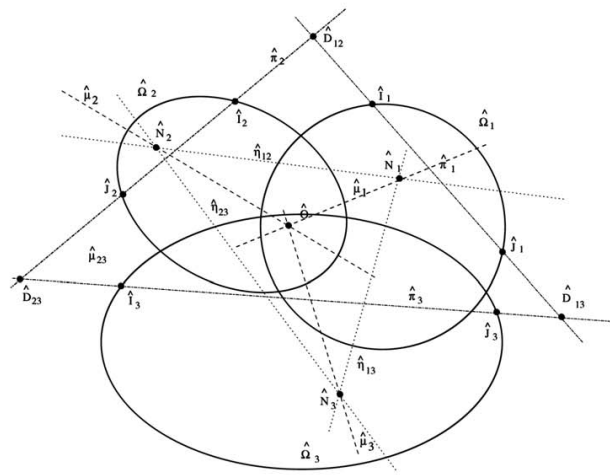

(b)

Fig. 5. Calibration of a central catadioptric system from the image of $K=3$ lines (Table 3). Geometric construction using the conic curves where the lines are projected: (a) Determining the image center $\hat{\mathbf{O}}$ and the absolute conic $\hat{\mathbf{\Omega}}_{\infty}$. (b) Computing the pose between the mirror and the camera and the shape parameter $\xi$.

points $\hat{\mathbf{I}}_{\mathbf{i}}, \hat{\mathbf{J}}_{\mathbf{i}}(i=1,2,3)$. Since these points lie on the image of the absolute conic (Proposition 2), then $\hat{\mathbf{\Omega}}_{\infty}$ can be estimated directly using the six points $\hat{\mathbf{I}}_{1}, \hat{\mathbf{J}}_{1}, \hat{\mathbf{I}}_{2}, \hat{\mathbf{J}}_{2}, \hat{\mathbf{I}}_{3}$, and $\hat{\mathbf{J}}_{3}$. Each pair of imaged lines has a line of normals associated with it, which is mapped on $\hat{\eta}_{\mathrm{ij}}$ in the catadioptric image plane (Fig. 5b). The line goes through the poles of $\hat{\boldsymbol{\mu}}_{i j}$ with respect to conics $\hat{\mathbf{\Omega}}_{\boldsymbol{i}}$ and $\hat{\boldsymbol{\Omega}}_{\boldsymbol{j}}$ (Proposition 7). Notice that points $\hat{\mathbf{N}}_{\mathrm{i}}$ and $\hat{\mathbf{N}}_{\mathrm{j}}$ both lie on $\hat{\boldsymbol{\eta}}_{\boldsymbol{i} \boldsymbol{j}}$. Thus, the pairs of lines $\hat{\boldsymbol{\eta}}_{\mathbf{1 2}}, \hat{\boldsymbol{\eta}}_{\mathbf{1 3}}$, $\hat{\boldsymbol{\eta}}_{12}, \hat{\boldsymbol{\eta}}_{23}$, and $\hat{\boldsymbol{\eta}}_{13}, \hat{\boldsymbol{\eta}}_{23}$ intersect in points $\hat{\mathbf{N}}_{1}, \hat{\mathbf{N}}_{2}$, and $\hat{\mathbf{N}}_{3}$. The determination of line $\hat{\boldsymbol{\mu}}_{\boldsymbol{i}}$ associated with the line image $\hat{\mathbf{\Omega}}_{\boldsymbol{i}}$, knowing both $\hat{\mathbf{O}}$ and $\hat{\mathbf{N}}_{\mathbf{i}}$, is trivial (Proposition 3). The locus of the line at infinity can be easily determined by considering that the poles of $\hat{\boldsymbol{\mu}}_{\mathbf{1}}$, $\hat{\boldsymbol{\mu}}_{2}$, and $\hat{\boldsymbol{\mu}}_{3}$ must lie in $\hat{\boldsymbol{\pi}}_{\infty}$ (Corollary 1 ). The value of $\xi$ is computed using the result of Proposition 4.

\section{Calibration of an Hyperbolic System Using THE IMAGE OF TWO LINES}

In [4], Geyer and Daniilidis advance the hypothesis that, while the calibration of a paracatadioptric camera requires at least three line images, the minimum number of lines to calibrate a hyperbolic/ elliptical system is two. This section uses a geometric construction to prove that a hyperbolic/elliptical system can be calibrated from the projection of two lines. We assume that both parameter $\xi$, depending on the mirror shape (Table 1), and line $\hat{\boldsymbol{\pi}}_{\infty}$ are known. This is a reasonable assumption for most commercial sensors, where $\hat{\boldsymbol{\pi}}_{\infty}=$ $(0,0,1)^{t}$ and $\mathbf{H}_{\mathbf{c}}$ is an affine transformation, because the rotation between the camera and the mirror is $\mathbf{R}_{\mathbf{c}}=\mathbf{I}$.

The geometric construction for calibration is summarized in Table 4 and illustrated in Fig. 4 . Line $\hat{\boldsymbol{\mu}}_{i j}$ is defined by the real points where conics $\hat{\boldsymbol{\Omega}}_{\boldsymbol{i}}$ and $\hat{\boldsymbol{\Omega}}_{\boldsymbol{j}}$ intersect. The result of Proposition 7 is used to compute the position of line $\hat{\boldsymbol{\eta}}_{\boldsymbol{i} \boldsymbol{j}}$. Since lines $\hat{\boldsymbol{\mu}}_{\boldsymbol{i} \boldsymbol{j}}, \hat{\boldsymbol{\eta}}_{\boldsymbol{i j}}$, and $\hat{\boldsymbol{\pi}}_{\infty}$ are known, then the computation of points $\hat{\mathbf{N}}_{\mathrm{ij}}, \hat{\mathbf{M}}_{\mathrm{ij}}$ and the respective conjugates $\hat{\mathbf{N}}_{\mathrm{ij}}^{*}, \hat{\mathbf{C}}_{\mathrm{ij}}$ lying on $\hat{\boldsymbol{\mu}}_{\boldsymbol{i}}$ is trivial. As discussed in Section 3.3, conics $\hat{\mathbf{\Omega}}_{\boldsymbol{i}}, \hat{\mathbf{\Omega}}_{\boldsymbol{j}}$ define a family of catadioptric line projections $\hat{\mathbf{\Omega}}$. The conic curve $\hat{\mathbf{\Omega}}_{\boldsymbol{i j}}$, depicted in Fig. 4 , is a particular realization of $\hat{\boldsymbol{\Omega}}$. It is the locus where $3 \mathrm{D}$ lines lying in the plane with normal direction $\overline{\mathbf{N}}_{\mathrm{ij}}=\overline{\boldsymbol{\mu}}_{\boldsymbol{i j}} \wedge \overline{\boldsymbol{\eta}}_{\boldsymbol{i} j}$ are projected. Notice that line $\hat{\boldsymbol{\mu}}_{\boldsymbol{i} j}$ passes through the image center Ô (Proposition 6). According to the result of Proposition 3, line $\hat{\boldsymbol{\mu}}_{i j}$ is the locus where the major axis of $\overline{\mathbf{\Omega}}_{i j}$ is mapped. Proposition 4 and (6) hold and the image center can be computed as described in Steps 6 and 7. The polar lines $\hat{\boldsymbol{\pi}}_{i}, \hat{\boldsymbol{\pi}}_{j}$ of the image center $\mathbf{O}$ intersect the respective conics at points $\hat{\mathbf{I}}_{\mathbf{i}}, \hat{\mathbf{J}}_{\mathbf{i}}, \hat{\mathbf{I}}_{\mathbf{j}}$, and $\hat{\mathbf{J}}_{\mathbf{j}}$ (see Fig. 4). From Proposition 2, it follows that these four points must lie on the image of the absolute conic $\hat{\mathbf{\Omega}}_{\infty}$. Moreover, $\hat{\mathbf{O}}$ and $\hat{\boldsymbol{\pi}}_{\infty}$ 
TABLE 4

Calibrating a Hyperbolic/Elliptical System from the Image of Two Lines

\begin{tabular}{|c|c|}
\hline Step 1 & Determine the catadioptric line images $\hat{\Omega}_{i}$ and $\hat{\Omega}_{j}$ using conic fitting techniques \\
\hline Step 2 & Compute the points $\hat{\mathbf{F}}_{\mathrm{ij}}, \hat{\mathbf{B}}_{\mathrm{ij}}$ where conics $\hat{\Omega}_{i}, \hat{\Omega}_{j}$ intersect and determine line $\hat{\mu}_{i j}=\hat{\mathbf{F}}_{\mathrm{ij}} \wedge \hat{\mathbf{B}}_{\mathrm{ij}}$ \\
\hline Step 3 & Obtain the line $\hat{\eta}_{i j}$ passing through the poles of $\hat{\mu}_{i j}$ with respect to conics $\hat{\Omega}_{i}$ and $\hat{\Omega}_{j}$ \\
\hline Step 4 & Compute the intersection points $\hat{\mathbf{M}}_{\mathbf{i j}}=\hat{\mu}_{i j} \wedge \hat{\boldsymbol{\pi}}_{\infty}$ and $\hat{\mathbf{N}}_{\mathrm{ij}}=\hat{\mu}_{i j} \wedge \hat{\eta}_{i j}$ \\
\hline Step 5 & $\begin{array}{l}\text { Obtain } \hat{\mathbf{C}}_{\mathbf{i j}} \text { and } \hat{\mathbf{N}}_{\mathbf{i j}}^{*} \text { such that both pairs of points }\left\{\hat{\mathbf{C}}_{\mathbf{i j}}, \hat{\mathbf{M}}_{\mathbf{i j}}\right\} \text { and }\left\{\hat{\mathbf{N}}_{\mathrm{ij}}^{*}, \hat{\mathbf{N}}_{\mathbf{i j}}\right\} \text { are harmonic conjugates } \\
\text { with respect to }\left\{\hat{\mathbf{F}}_{\mathrm{ij}}, \hat{\mathbf{B}}_{\mathbf{i j}}\right\}\left(\hat{\mathbf{C}}_{\mathbf{i j}}=\hat{\mu}_{i j} \wedge\left(\hat{\mathbf{\Omega}}_{i} \hat{\mathbf{M}}_{\mathbf{i j}}\right)=\hat{\boldsymbol{\mu}}_{i j} \wedge\left(\hat{\mathbf{\Omega}}_{j} \hat{\mathbf{M}}_{\mathbf{i j}}\right) \text { and } \hat{\mathbf{N}}_{\mathbf{i j}}^{*}=\hat{\mathbf{\Omega}}_{i}^{*} \cdot \hat{\boldsymbol{\eta}}_{i j}=\hat{\mathbf{\Omega}}_{j}^{*} \cdot \hat{\boldsymbol{\eta}}_{i j}\right) \text {. }\end{array}$ \\
\hline Step 6 & Determine the cross-ratio $\left\{\hat{N}_{i j}^{*}, \hat{\mathbf{N}}_{i j} ; \hat{\mathbf{M}}_{\mathbf{i j}}, \hat{\mathbf{C}}_{\mathbf{i j}}\right\}$ and compute $\left\{\hat{\mathbf{C}}_{\mathbf{i j}}, \hat{\mathbf{N}}_{\mathbf{i j}} ; \hat{\mathbf{M}}_{\mathbf{i j}}, \hat{\mathbf{O}}_{\mathbf{i j}}\right\}$ using equation 6 \\
\hline Step 7 & Determine the image center $\hat{\mathbf{O}}$ from points $\hat{\mathbf{C}}_{\mathbf{i j}}, \hat{\mathbf{N}}_{\mathbf{i} \mathbf{j}}, \hat{\mathbf{M}}_{\mathbf{i j}}$ and the value of $\left\{\hat{\mathbf{C}}_{\mathbf{i j}}, \hat{\mathbf{N}}_{\mathbf{i j}} ; \hat{\mathbf{M}}_{\mathbf{i j}}, \hat{\mathbf{O}}_{\mathbf{i j}}\right\}$ \\
\hline Step 8 & Obtain the polar lines $\hat{\pi}_{i}, \hat{\pi}_{j}$ of the image center $\hat{\mathbf{O}}$ with respect to conics $\hat{\Omega}_{i}, \hat{\Omega}_{j}$ \\
\hline Step 9 & Determine the intersection points $\hat{\mathbf{I}}_{\mathbf{i}}, \hat{\mathbf{J}}_{\mathbf{i}}$ of $\hat{\pi}_{i}$ with $\hat{\Omega}_{i}$, and $\hat{\mathbf{I}}_{\mathbf{j}}, \hat{\mathbf{J}}_{\mathbf{j}}$ of $\hat{\pi}_{j}$ with $\hat{\Omega}_{j}$ \\
\hline Step 10 & $\begin{array}{l}\text { Estimate the absolute conic knowing that points } \hat{\mathbf{I}}_{\mathbf{i}}, \hat{\mathbf{J}}_{\mathbf{i}}, \hat{\mathbf{I}}_{\mathbf{j}} \text { and } \hat{\mathbf{J}}_{\mathbf{j}} \text { lie in the conic and that } \hat{\mathbf{O}}_{\mathbf{i}} \text { and } \hat{\boldsymbol{\pi}}_{\infty} \\
\text { are pole/polar with respect to } \hat{\mathbf{\Omega}}_{\infty}\end{array}$ \\
\hline Step 11 & Compute transformation $\mathbf{H}_{\mathrm{c}}$ from the Cholesky decomposition of $\hat{\boldsymbol{\Omega}}_{\infty}$. \\
\hline
\end{tabular}

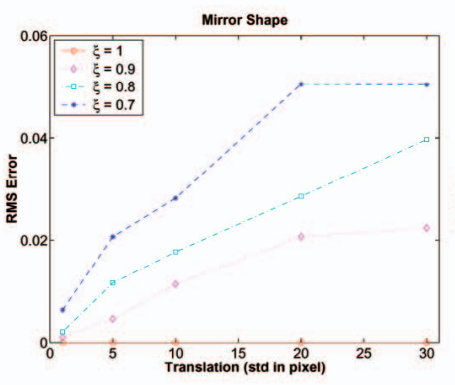

(a)

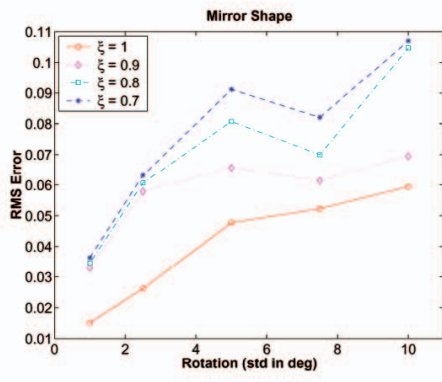

(b)

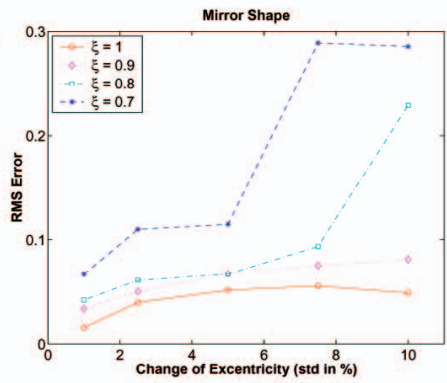

(c)

Fig. 6. Calibration of a general central catadioptric system using $K=3$ line images. The graphics show the sensitivity of the estimation of the mirror shape $\xi$ to errors on the conic curves. In (a) and (b), the conic curves are, respectively, translated and rotated by a certain amount. Graphic (c) shows the effect of changing the eccentricity.

are pole/polar with respect to $\hat{\mathbf{\Omega}}_{\infty}$. The absolute conic is estimated using these five constraints.

The geometric construction of Table 4 cannot be used to calibrate a paracatadioptric camera. Since points $\hat{\mathbf{N}}_{\mathrm{ij}}$ and $\hat{\mathbf{M}}_{\mathrm{ij}}$ are coincident with points $\hat{\mathbf{C}}_{\mathrm{ij}}$ and $\hat{\mathbf{N}}_{\mathrm{ij}}^{*}$, respectively (Proposition 5), then the cross$\operatorname{ratios}\left\{\hat{\mathbf{N}}_{\mathrm{ij}}^{*}, \hat{\mathbf{N}}_{\mathrm{ij}} ; \hat{\mathbf{M}}_{\mathrm{ij}}, \hat{\mathbf{C}}_{\mathrm{ij}}\right\}$ and $\left\{\hat{\mathbf{C}}_{\mathrm{ij}}, \hat{\mathbf{N}}_{\mathrm{ij}} ; \hat{\mathbf{M}}_{\mathrm{ij}}, \hat{\mathbf{O}}_{\mathrm{ij}}\right\}$ are 0 and 1 and (6) can no longer be used to determine the image center $\mathbf{O}$. It seems that the projection of two lines does not provide enough information to calibrate the paracatadioptric camera. This can be interpreted as follows: The goal is to estimate the image of the absolute conic $\hat{\mathbf{\Omega}}_{\infty}$, which has five unknown parameters. Since each line introduces two additional unknowns, by considering two line images we have a total of nine parameters to determine. For the hyperbolic/elliptical situation, each line is projected into a conic curve with five degrees of freedom. Thus, two line images provide 10 constraints and the system can be calibrated. However, for the paracatadioptric sensor, the line image always goes through the locus of the circular points [10]. The first line image provides five constraints, while any additional line only gives three constrains. The calibration from two line images is an underdetermined problem because there are only eight constraints for nine unknowns.

\section{EXPERIMENTAL RESULTS}

Section 4 gives a geometric construction for calibrating any central catadioptric system using three or more lines. The calibration is 100 percent accurate whenever the conics corresponding to the catadioptric projection of lines are determined with zero error. In practice, there are always errors in the estimation of the conic curves that propagate and cause errors in the calibration.

\subsection{Sensitivity Analysis}

Consider a catadioptric sensor with a certain parameter $\xi$ and predefined intrinsic matrix $\mathbf{H}_{\mathbf{c}}$. Synthetic images of a random set of $K=3$ lines are generated by choosing three normal vectors $\mathbf{n}$ from a uniform distribution on the sphere. To each normal $\mathbf{n}$ corresponds a conic curve $\hat{\mathbf{\Omega}}$ in the catadioptric image plane (Fig. 2 and (4) and (5)). The method summarized in Table 3 can be applied to the synthetic image. In order to evaluate the stability of the geometric construction, the catadioptric projection of each line is artificially distorted before applying the method. In Fig. $6 a$, each conic curve $\hat{\mathbf{\Omega}}$ is independently translated by a random $2 \mathrm{D}$ vector. The translation direction is determined assuming a uniform distribution, while the amplitude is chosen from a Gaussian distribution with zero mean and standard deviation $\sigma$ (X axis in the graphics). The system is calibrated using the distorted images of lines. The results are compared with the ground truth and the RMS error is computed over 300 runs of each experiment. The other two graphics show the sensitivity to different types of distortion. In Fig. 6 b, each curve $\hat{\boldsymbol{\Omega}}$ is rotated around the center by an angle $\theta$, while, in Fig. 6c, the distortion is caused by a change in percentage $e$ on the eccentricity of each conic. Both $\theta$ and $e$ are randomly chosen from a Gaussian distribution with zero mean. As expected, the accuracy of the calibration decreases when the magnitude of distortion increases. According to the experiments, the method appears to be more robust when used to calibrate a parabolic sensor.

\subsection{Experiments Using Real Images}

Consider the image in Fig. 7a, acquired by an uncalibrated paracatadioptric camera, where several lines in the scene are 


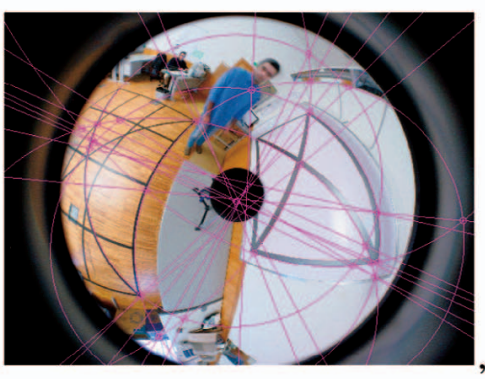

(a)

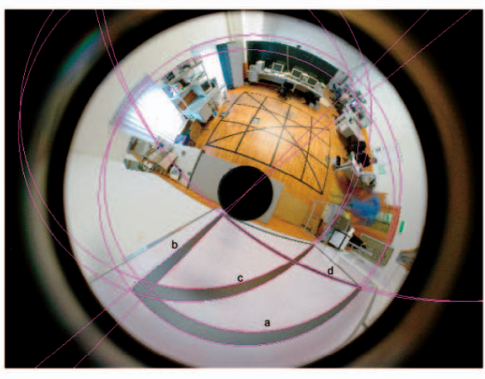

(b)

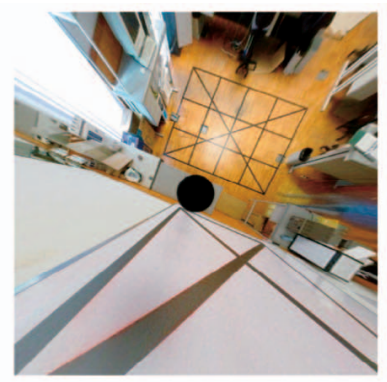

(c)

Fig. 7. Experiments using real images. In (a), a set of line projections is estimated using conventional conic fitting techniques. In (b), several pairs of parallel lines are estimated and the angles between them are computed. In (c), the parallel lines are determined after perspective rectification and the angles are computed.

TABLE 5

Recovering Angles between Pairs of Parallel Lines

\begin{tabular}{|l||llllll||ll||}
\hline & a-b & a-c & a-d & b-c & b-d & c-d & Mean & Std \\
\hline G. Truth & $90^{\circ}$ & $30^{\circ}$ & $30^{\circ}$ & $60^{\circ}$ & $60^{\circ}$ & $60^{\circ}$ & & \\
\hline Error PER & $0.34^{\circ}$ & $6.64^{\circ}$ & $0.49^{\circ}$ & $2.40^{\circ}$ & $13.71^{\circ}$ & $16.01^{\circ}$ & $\mathbf{6 . 6 2}^{\circ}$ & $\mathbf{6 . 8 5}^{\circ}$ \\
Error CAT & $0.85^{\circ}$ & $2.05^{\circ}$ & $1.82^{\circ}$ & $2.99^{\circ}$ & $1.04^{\circ}$ & $3.87^{\circ}$ & $\mathbf{2 . 1 0}^{\circ}$ & $\mathbf{3 . 8 7}^{\circ}$ \\
\hline
\end{tabular}

projected on a set of conic curves. The curves are estimated by applying a conventional conic fitting technique [11]. According to Proposition 6, the lines passing through the intersection points of any two conics must meet in a single point (the image center). This property is clearly not satisfied, which means that the conic curves are not correctly estimated. The problem is that, in general, only a small arc of the curve is visible in the image, and conventional conic fitting algorithms tend to fail [11]. The estimation of the conics corresponding to the catadioptric projection of lines is beyond the scope of the present work. In [12], we propose a method that uses specific constraints to cope with the ambiguity introduced by the partial occlusion. Extensive experimental results show that the proposed conic fitting algorithm combined with the geometric construction of Table 3 outperforms the calibration methods currently available.

The usefulness of the properties of catadioptric lines introduced in Section 3 is not limited to the geometric constructions to recover the calibration parameters. Fig. $7 \mathrm{~b}$ shows a paracatadioptric image of four pairs of parallel lines lying in a plane in the scene. The sensor was previously calibrated using the method summarized in Table 3 [12]. Since the calibration is known, the catadioptric image can be rectified into a conventional perspective as shown in Fig. 7c. The angles between parallel pairs can be easily computed by estimating the lines and determining the corresponding vanishing points. An alternative way of determining these angles is to use the result of Corollary 2. In this case, the line images are estimated directly in the catadioptric image plane using the algorithm proposed in [10]. Table 5 shows the errors in the estimation of the angles in both cases. The method of computing the angles directly in the catadioptric images (CAT) outperforms the approach of making the measurement in the rectified perspective (PER). The poorer performance of PER is explained by the statistical bias introduced during the rectification process (for details, see [8]).

\section{Closure}

This paper introduces a geometric theory for central catadioptric projection of lines. A set of projective invariant properties has been systematically presented. These properties enable a better understanding of the geometry of central catadioptric images and are useful for both calibration and reconstruction purposes. In [5], it is shown that a paracatadioptric sensor can be calibrated using an image of three lines. We have generalized this result and proven that any central catadioptric system can be fully calibrated using a minimum of three lines. Moreover, it has also been shown that, if the system is hyperbolic/elliptical, then two line images are enough to recover the system parameters.

\section{REFERENCES}

[1] P. Sturm, "A Method for 3D Reconstruction of Piecewise Planar Objects from Single Panoramic Images," Proc. IEEE Workshop Omnidirectional Vision, pp. 119-126, June 2000.

[2] J.P. Barreto, F. Martin, and R. Horaud, "Visual Servoing/Tracking Using Central Catadioptric Images," Proc. Eighth Int'l Symp. Experimental Robotics, July 2002.

[3] T. Svoboda and T. Pajdla, "Epipolar Geometry for Central Catadioptric Cameras," Int'l J. Computer Vision, vol. 49, no. 1, pp. 23-37, Aug. 2002.

[4] C. Geyer and K. Daniilidis, "An Unifying Theory for Central Panoramic Systems and Pratical Implications," Proc. European Conf. Computer Vision, pp. 445-461, 2000.

[5] C. Geyer and K. Daniilidis, "Paracatadioptric Camera Calibration," IEEE Trans. Pattern Analysis and Machine Intelligence, vol. 24, no. 4, Apr. 2002.

[6] D. Liebowitz, "Camera Calibration and Reconstruction of Geometry from Images," PhD thesis, Univ. of Oxford, 2001.

[7] S. Baker and S. Nayar, "A Theory of Catadioptric Image Formation," Proc. IEEE Int'l Conf. Computer Vision, pp. 35-42, 1998.

[8] J.P. Barreto, "General Central Projection Systems: Modeling, Calibration and Visual Servoing," PhD thesis, Univ. of Coimbra, 2003.

[9] J.G. Semple and G.T. Kneebone, Algebraic Projective Geometry. Clarendon Press, 1998.

[10] J.P. Barreto and H. Araujo, "Direct Least Square Fitting of Paracatadioptric Line Images," Proc. Workshop Omnidirectional Vision and Camera Networks, June 2003.

[11] Z. Zhang, "Parameter Estimation Techniques: A Tutorial with Application to Conic Fitting," INRIA Raport de Recherche n 2676, Oct. 1995.

[12] J.P. Barreto and H. Araujo, "Paracatadioptric Camera Calibration Using Lines," Proc. IEEE Int'l Conf. Computer Vision, Oct. 2003.

$\triangleright$ For more information on this or any other computing topic, please visit our Digital Library at www.computer.org/publications/dlib. 